\title{
Complex Boundary Value Problems of Nonlinear Differential Equations: Theory, Computational Methods, and Applications
}

\author{
Xinguang Zhang, ${ }^{1}$ Yong Hong Wu, ${ }^{2}$ Dragoş-Pătru Covei, ${ }^{3}$ and Xinan Hao ${ }^{4}$ \\ ${ }^{1}$ School of Mathematical and Informational Sciences, Yantai University, Shandong, Yantai 264005, China \\ ${ }^{2}$ Department of Mathematics and Statistics, Curtin University of Technology, Perth, WA 6845, Australia \\ ${ }^{3}$ Department of Development, Constantin Brancusi University of Târgu-Jiu, Grivitei Street, No. 1, Târgu-Jiu 210131, Romania \\ ${ }^{4}$ School of Mathematical Sciences, Qufu Normal University, Shandong, Qufu 273165, China
}

Correspondence should be addressed to Xinguang Zhang; zxg123242@163.com

Received 18 April 2013; Accepted 18 April 2013

Copyright (C) 2013 Xinguang Zhang et al. This is an open access article distributed under the Creative Commons Attribution License, which permits unrestricted use, distribution, and reproduction in any medium, provided the original work is properly cited.

This special issue focuses on the theme Complex Boundary Value Problems of Nonlinear Differential Equations: Theory, Computational Methods, and Applications which plays a tremendous role in the study and control of the real-world systems and the development of new technologies. The special issue aim is to present some of the recent developments in this field.

The issue contains 36 papers selected through a peerreviewed process. Many of the papers focus on the study of problems that have attracted attention from the research community for many years and involve the development of innovative ideas and in-depth scientific approaches. In the following, we briefly review each of the papers highlighting and the key contributions of them.

Through this special issue, we also hope to open the opportunity for the journal readers to make comments on the works presented.

In the paper titled " $A$ finite element method for multi-term time-space riesz fractional advection-diffusion equations in finite domain," the authors present an effective finite element method for multiterm time-space Riesz fractional advectiondiffusion equations. Some numerical examples are also given to match with the main conclusions.

In the paper titled "Nonlocal problem for fractional evolution equations of mixed type with the measure of noncompactness," the authors study the existence of mild solutions and positive mild solutions for a general class of semilinear fractional evolution equations of mixed type with nonlocal conditions on infinite dimensional Banach spaces, by utilizing a new estimation technique of the measure of noncompactness and a new fixed-point theorem with respect to convex-condensing operator.

In the paper titled "A robust weak taylor approximation scheme for solutions of jump-diffusion stochastic delay differential Equations," the authors construct a Taylor approximation scheme and examine the convergence of the method in a weak sense. The analysis and numerical results show that the proposed scheme of high order is effective and efficient for the solution of jump-diffusion stochastic delay differential equations.

In the paper titled "Research on internal layout optimization of logistics node under the conditions of complex terrain based on computer vision and geographical simulation system," the authors proposed an internal layout optimization nonlinear mathematical data model of logistics node. By borrowing ideas from construction algorithm, initial solution is made by taking the advantage of generating initial solution. By adopting the ideas of improved algorithm, research on logistics node layout optimization is conducted on the combination of genetic algorithm, simulated annealing algorithm, and multiagent technology so as to realize large-scale logistics node internal layout optimization under the conditions of complex terrain and multiple constraints.

In the paper titled "A contraction fixed point theorem in partially ordered metric spaces and application to fractional differential equations," the authors' objective is to generalize a 
fixed-point result, from the literature. As an application of this result the reader can find the existence and uniqueness for a positive solution of a multipoint boundary value problem with fractional derivatives.

In the paper titled "Positive solutions for fractional differential equations from real estate asset securitization via new fixed point theorem," the authors obtained some optimize scheme for traditional risk control process in the analysis of the existence and uniqueness of the solution and scientific numerical calculation of the solution. The author's results can be applied to the forefront of research of real estate asset securitization.

In the paper titled "Nontrivial solution of fractional differential system involving riemann-stieltjes integral condition," the author obtained the existence and uniqueness of nontrivial solutions for a class of fractional differential system involving Riemann-Stieltjes integral condition, under some suitable hypotheses. The proof is based on a Leray-Schauder nonlinear alternative and Banach contraction mapping principle.

In the paper titled "Travelling wave solutions to the benney-luke and the higher-order improved boussinesq equations of sobolev type," the authors obtained some travelling wave solutions of two well-known nonlinear Sobolev-type partial differential equations, namely, the Benney-Luke equation and the higher-order improved Boussinesq equation, merely by using the tanh-coth method. It is remarkable that equations of Sobolev type describe many physical phenomena and that there are many studies concerned with such equations (among them the stability analysis, Cauchy problem, existence and analyticity of solutions, and travelling wave solutions can be mentioned).

In the paper titled "Exponential stability of impulsive delay differential equations," the authors investigate the exponential stability of a class of impulsive delay differential equations.

In the paper titled "A Combined mathematical treatment for a special automatic music transcription system," the authors present some interesting results about the mathematical treatment for a special automatic music transcription system. The algorithm obtained here reduces the dimension by PCA and selects candidates first by human auditory model and harmonic structures of notes. It changes the multiple-F0 estimation question into a mathematical problem and solves it in a mathematical way.

In the paper titled "A Godunov-mixed finite element method on changing meshes for the nonlinear Sobolev equations," the author presents a Godunov-mixed finite-element method on changing meshes to simulate the nonlinear Sobolev equations. Moreover, a numerical experiment is given to illustrate the efficiency of the method.

In the paper titled "Entire blow-up solutions of semilinear elliptic systems with quadratic gradient terms," the authors' objective is to give an answer to an open problem from the literature, regarding the existence of solutions to a problem with quadratic gradient terms. The method given here is based especially on the approximation theory.

In the paper titled "The global weak solution for a generalized Camassa-Holm equation," the author's objective is to establish global weak solutions for a generalized CamassaHolm equation.

In the paper titled "Computation of Domain of the attraction for suboptimal immunity epidemic models using the maximal lyapunov function method," the authors deal with the estimation of the domain of attraction for suboptimal immunity epidemic models. They established a procedure to determine the maximal Lyapunov function in the form of rational functions. Here are especially considered the suboptimal immunity epidemic models with saturated treatment rate and nonlinear incidence rate. We can see that the suboptimal immunity models are more realistic to explain the microparasite infection diseases such as Pertussis and Influenza A.

In the paper titled "Variational image denoising approach with diffusion porous media flow," the author's goal was to develop a novel anisotropic diffusion based image denoising approach which outperforms the influential scheme of Perona-Malik by removing a greater amount of noise, reduces the staircasing effect, and preserves better image edges.

In the paper titled "Eigenvalue problem of nonlinear semipositone higher order fractional differential equations," the authors deal with the eigenvalue interval for existence of positive solutions to the higher-order fractional differential equation when the nonlinearity may be negative. This type of differential equations is called semipositone problem which arises in many interesting applications. For example, the semipositone differential equation can be derived from chemical reactor theory, design of suspension bridges, combustion, and management of natural resources.

In the paper titled "A finite difference scheme for compressible miscible displacement flow in porous media on grids with local refinement in time," the author constructed and studied finite difference schemes on grids with local refinement in time for two-dimensional compressible miscible displacement flow in porous media.

In the paper titled "General formulation of second-order semi-lagrangian methods for convection-diffusion problems," the authors obtained general formulation of the second-order semi-Lagrangian methods for convection-dominated diffusion problems. The relationship between semi-Lagrangian methods and the modified method of characteristic is also addressed.

In the paper titled "Existence results For solutions of integral boundary value problems on time scales," the authors obtained sufficient conditions for the existence of solutions for a class of integral boundary problems on time scale. The main instrument here is the Schauder fixed-point theorem in a cone. existence result for the considered problem is also given by the method of upper and lower solutions.

In the paper titled "Jensen's inequality for generalized peng's g-expectations and its applications," the author extends some existing results about Jensen's inequality for generalized Peng's g-expectations. Furthermore, some applications of Jensen's inequality for generalized Peng's $g$-expectations can be found in the work.

In the paper titled "Adaptive finite element method for optimal control problem governed by linear quasiparabolic 
integrodifferential equations," the authors describe semidiscrete adaptive finite-element approximation schemes for a constrained optimal control problem governed by linear quasiparabolic integrodifferential equations.

In the paper titled "Logarithmically improved regularity criteria for a fluid system with the linear Soret effect," the authors obtained logarithmically improved regularity criterion in the $\mathrm{BMO}$ space for a 3D fluid system with the linear Soret effect.

In the paper titled "Bifurcations of a homoclinic orbit to saddle-center in reversible systems," by establishing a new kind of local moving frame along the primary homoclinic orbit and using the Melnikov functions, the authors obtained the existence and nonexistence of 1-homoclinic orbit and 1periodic orbit, including symmetric 1-homoclinic orbit and 1-periodic orbit, and their corresponding codimension 1 or codimension 3 surfaces, for a reversible system considered.

In the paper titled "Uniqueness and asymptotic behavior of positive solutions for a fractional-order integral boundary value problem," the authors obtained the existence, uniqueness, and asymptotic behavior of positive solutions for a singular nonlocal integral boundary value problem for fractional differential model arising from porous media, electromagnetic and signal processing of wireless communication system.

In the paper titled "Existence of positive solutions for multipoint boundary value problems on infinite intervals in Banach spaces," the authors used the Mönch fixed-point theorem to give the existence of a positive solutions of a class of second-order nonlinear singular differential equations with multipoint boundary value conditions on an infinite interval in Banach spaces.

In the paper titled "Multigrid discretization and iterative algorithm for mixed variational formulation of the eigenvalue problem of electric field," the authors discussed highly finiteelement algorithms for the eigenvalue problem of electric field.

In the paper titled "A two-grid method for finite element solutions of nonlinear parabolic equations," the authors presented and discussed a two-grid method for a finite-element approximation to a nonlinear parabolic equation in two space dimensions.

In the paper titled "Valuation of the prepayment option of a perpetual corporate loan," the authors deal with a concrete model problem from the economy. The main idea of the paper is detailed here: the economy needs investments in order to function correctly, and this function is mainly performed by banks. The banks lend money to firms in exchange of periodic payments of interest (and the final payment of the borrowed amount). These agreements, called "corporate loans," may feature some particular clauses such as the possibility for the borrower to pay in advance the loan; such a clause is called "prepayment." In order to eliminate possible risks related to fluctuations in the interest rates, the banks hedge their exposure to such risks for any corporate loan. However, when the client prepays, this may imply some new costs to the bank. The authors' contribution proposes a mathematical model to assess the costs related to the prepayment of corporate loans. The mathematical model is first a Markov-modulated CIR stochastic process. Then, a system of partial differential variational inequalities is derived. The numerical computation of the solution needs to identify the (unknown) boundary between the domain where some partial differential operator is zero (the "continuity" domain) and the domain where the operator is only positive (the "exercise" region). The mathematical study of this work allows, among others, to certify that there is exactly one such boundary and thus precisely one continuity domain and precisely one exercise domain. We also remark that the authors compare the results with banking practice and show that the model is coherent and it should be considered when estimating the risks related to the prepayment of a corporate loan.

In the paper titled "Stability and convergence of an effective finite element method for multiterm fractional partial differential equations," the authors are concerned with an effective finite-element method for an initial and boundary value problem of the multi-term fractional partial differential equations. Furthermore, the stability and convergence for numerical methods are discussed. Finally, the numerical examples are given to match with the main conclusions and show the effectiveness of the method.

In the paper titled "Bifurcations of a ratio-dependent Holling-Tanner system with refuge and constant harvesting," the authors investigated the bifurcation properties of a predator prey system with refuge and constant harvesting.

In the paper titled " $h$-Stability of linear matrix differential systems," the authors investigate the stability problem of linear matrix differential systems by employing the Kronecker product of matrices and give some sufficient conditions of $h$ stability for linear matrix system and its associated perturbed system by using the Kronecker product of matrices.

In the paper titled "Uniqueness in inverse electromagnetic conductive scattering by penetrable and inhomogeneous obstacles with a Lipschitz boundary," the author deals with the problem of scattering of time-harmonic electromagnetic waves by a penetrable, inhomogeneous Lipschitz obstacle covered with a thin layer of high conductivity.

In the paper titled "Multiple positive solutions of a singular semipositone integral boundary value problem for fractional q-derivatives equation," the authors investigate a class of singular semipositone integral boundary value problem for fractional $q$-derivatives equations and obtain sufficient conditions for the existence of at least two and at least three positive solutions, under suitable conditions for each of the existence of solutions cases.

In the paper titled "Numerical method for a Markovmodulated risk model with two-sided jumps," the purpose is to present some numerical results on the Gerber-Shiu function for the Markov-modulated diffusion risk model with arbitrary upward and downward jumps.

In the paper titled "Roles of weight functions to a nonlocal porous medium equation with inner absorption and nonlocal boundary condition," the authors deal with sufficient conditions of global and blow-up solutions for an initial boundary value problem for a nonlocal porous medium equation with inner absorption and weighted nonlocal boundary condition. 
In the paper titled "The $H^{1}(R)$ space global weak solutions to the weakly dissipative Camassa-Holm equation," the authors' aim is to study the existence of global weak solutions of the Cauchy problem for a generalized Camassa-Holm equation with a dissipative term. It is remarkable that up to this work, there have been no global existence results for weak solutions to the generalized Camassa-Holm equation considered.

\title{
Acknowledgment
}

Here, we would like to thank all the authors and reviewers of the papers for their excellent contributions.

\author{
Xinguang Zhang \\ Yong Hong Wu \\ Dragoş-Pătru Covei \\ Xinan Hao
}




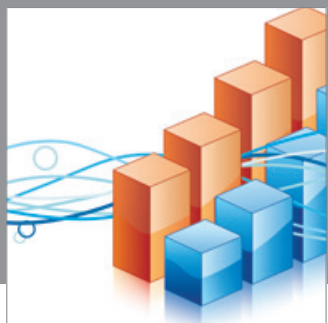

Advances in

Operations Research

mansans

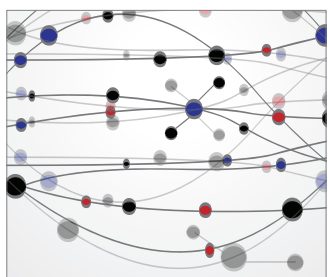

The Scientific World Journal
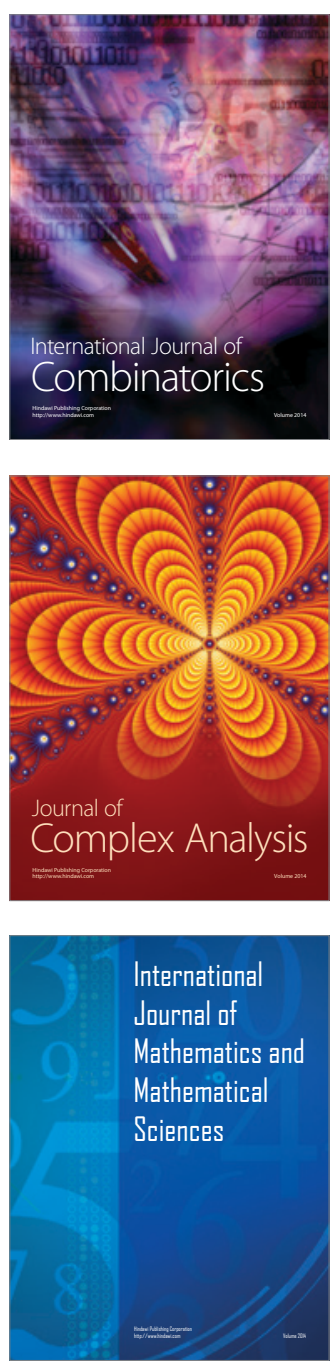
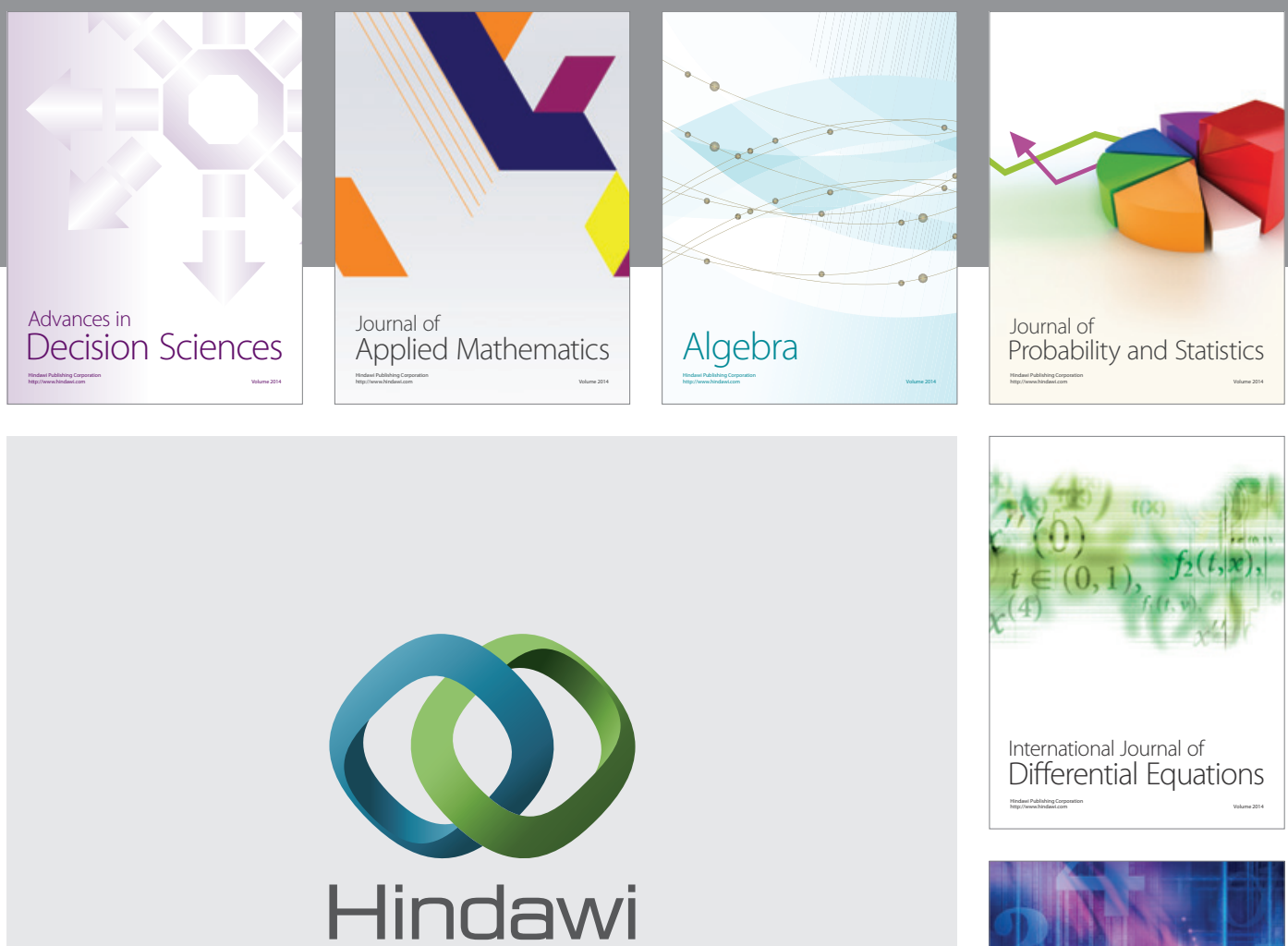

Submit your manuscripts at http://www.hindawi.com
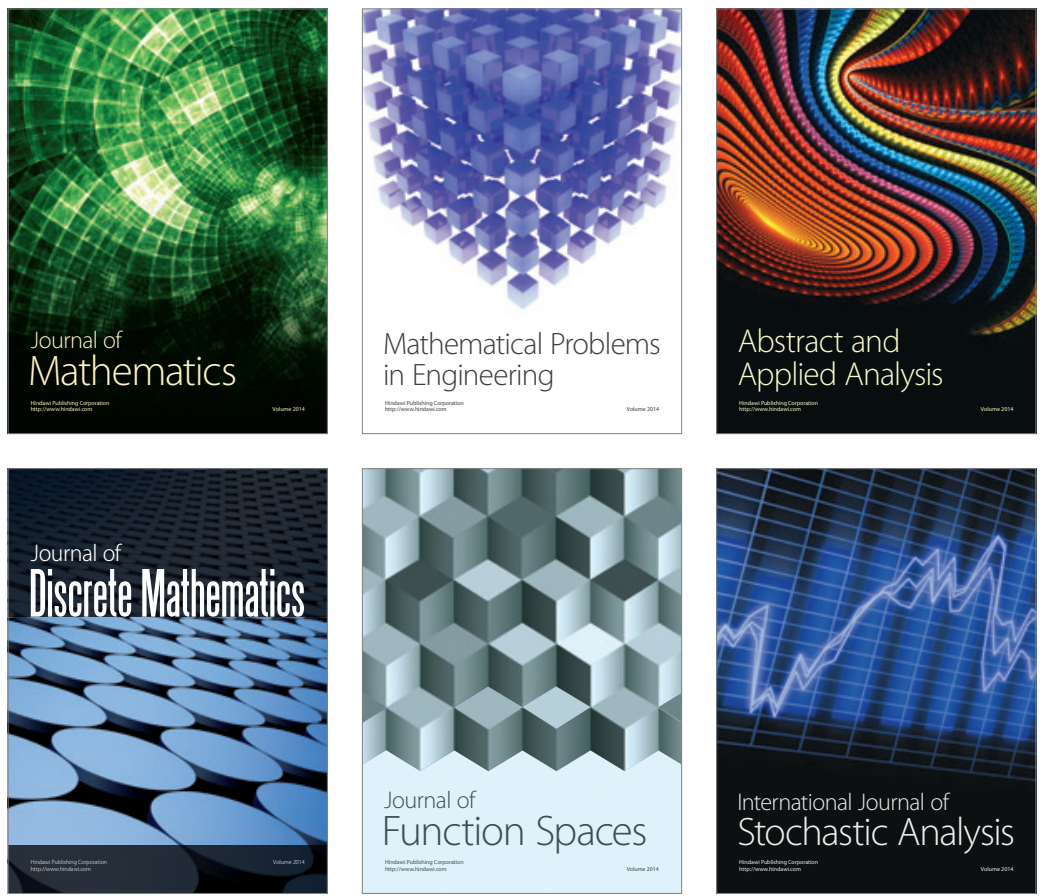

Journal of

Function Spaces

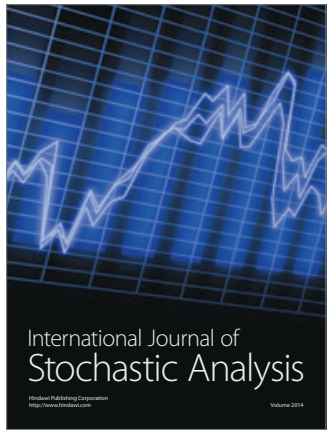

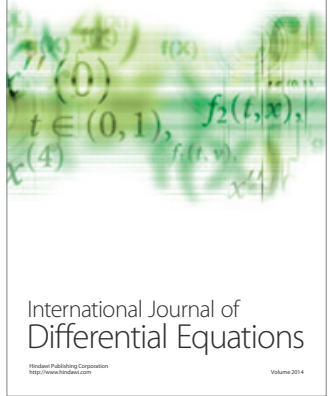
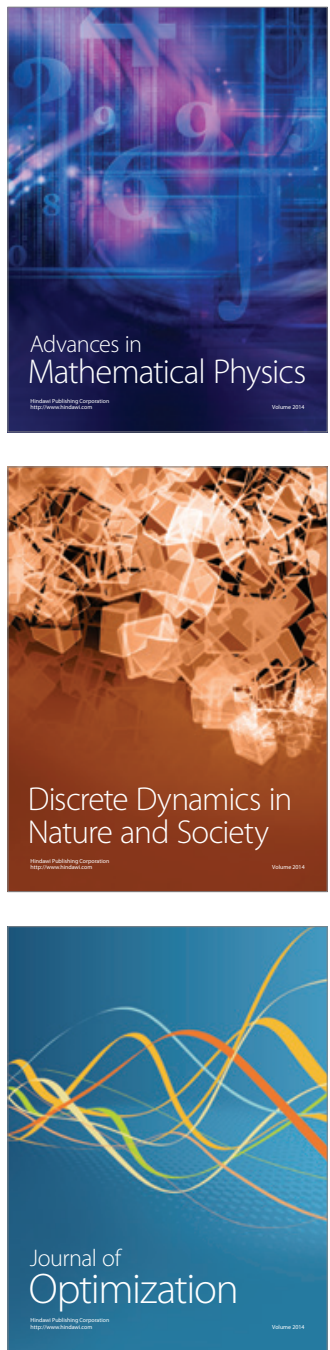